\title{
Pharmacokinetics of Cycloserine in Rats by HPLC-MS/MS
}

\section{Liping Yan, An Xie, Zhuo Wang, Wenjing Zhang, Yi Huang and Heping Xiao*}

Department of Tuberculosis, Shanghai Pulmonary Hospital, Tongji University School of Medicine, 507 Zhengmin Rd., Shanghai 200433, People's Republic of China

\begin{abstract}
Objective To investigate the pharmacokinetics of cycloserine in healthy rat blood and lung tissues. Method Healthy rat blood and lung tissues were sampled in vivo by microdialysis sampling technique simultaneously. The concentrations of cycloserine in both blood and lung tissues were measured by high performance liquid chromatography mass spectrometry. All data were analyzed by Win Nonlin software. Results:The maximum concentration of free cycloserine in blood and lung tissue were $(10.61 \pm 2.42) \mathrm{mg} / \mathrm{L}$ and $(1.53 \pm 1.71) \mathrm{mg} / \mathrm{L}$ at $1 \mathrm{~h}$. And then both continued to decline. After administration the concentrations of free cycloserine in the blood has been higher than the concentrations in lung tissues. The area under the concentration curve (AUC) of free cycloserine was (33.53 \pm 6.51$) \mathrm{h} \cdot \mathrm{mg}-1 \cdot \mathrm{L}-1 \mathrm{in}$ blood, and $(4.49 \pm 2.08) \mathrm{h} \cdot \mathrm{mg}-1 \cdot \mathrm{L}-1$ in lung tissues.
\end{abstract}

Conclusion Microdialysis sampling technique combined with high performance liquid chromatography mass spectrometry / mass spectrometry can be accurately and objectively reflect the drug in the blood and tissues of the pharmacokinetic characteristics of cycloserine. Concentrations in lung tissues were significantly lower than concentrations in blood.

Keywords: Cycloserine; Microdialysis; Pharmacokinetics; Pharmacodynamic; High performance liquid chromatographymass spectrometry / mass spectrometry

\section{Introduction}

Tuberculosis (TB) ranks as the second leading cause of death from an infectious disease worldwide [1]. Globally, $3.5 \%$ of new and $20.5 \%$ of previously treated TB cases was estimated to have Multidrug-resistant TB (MDR-TB).Advances in the fields of therapeutic medicine have had a less than optimal effect. In 2013 , overall only $48 \%$ of patientswith MDR-TB were successfully treated [1]. Cycloserine is a new secondline anti-tuberculosis drug and mainly used in combination with other drugs for the treatment of MDR-TB. Cycloserine blocks the synthesis of adhesive peptide of bacterial cell wall by inhibiting the function of D- alanine racemase (Alr) and synthetase (Ddl) [2]. Therefore it has strong killing effect against Mycobacterium tuberculosis (MTB) and Non-tuberculosis mycobacteria (NTM). Cycloserine also has the characteristics of good capability of penetrating in tissues and light injury to liver [3]. In addition, cycloserine resistance is fairly low [4]. Therefore, WHO recommends the regimen containing cycloserine for the treatment of MDR-TB [5]. Currently, the concept of the target site concentration as a direct link to therapeutic drug effects and direct target tissue concentration measurements as a means of optimizing individual drug therapy is receiving much attention [6]. Studies on pharmacokinetics of cycloserine in blood and lung tissue can provide reference for clinical medication. Unfortunately, we have not found many relevant reports. To address this need, we conducted this study to investigate the pharmacokinetics of cycloserine in healthy rats blood and lung tissues by Microdialysis and High performance liquid chromatography mass spectrometry / mass spectrometry (HPL-MS/ MS) method.

Testing drug concentration in lung tissue traditionally use whole lung tissue homogenate, saliva, respiratory secretions, pleural effusion, epithelial lining fluid especially bronchial alveolar lavage (BAL) fluid sampling [5]. But these methods can not accurately reflect the metabolism of free drug in lung interstitial fluid. Microdialysis is an emerging biological sampling technique, mainly using the principle of semipermeable membrane having permeability to small molecular compounds and material diffusion along a concentration gradient. Protein bound drugs at the testing site is trapped in dialysis membrane outside probe and the detected sample was free drug. Combined with the probe dialysis rate, sampling the material of extracellular fluid (ECF), can calculate the corresponding drug concentration in extracellular fluid of the target tissue in the same individual at dense time point [7]. Research shows that the microdialysis technique is a safe and effective way of assessing the pharmacokinetics of antimicrobial agents in lung tissue [8]. In vivo, real-time and continuous sampling can be performed without disrupting the normal physiological process. It also has the advantages of small amount of sampling, small interference of organism balance and direct determination of pulmonary free small molecule concentration. These have important significance in the research of drugs. Sample analysis in this study use HPL-MS/ MSmethod. Compared with the previous simple high performance liquid chromatography, it not only has the liquid separation powerful phase analysis ability, but also has the mass sensitive identification and structure analysis ability. HPLC-MS/MS technology has advantages of detection sample diversity, repeatable quantitative analysis, sufficient sensitivity and selectivity, rapid analysis and convenient. It also can analysis the complex molecular structure of body fluid.

\section{Materials and Methods}

\section{Animals}

Total six Sprague-Dawley (SD) male rats (60d old) weighting 220 $250 \mathrm{~g}$ were provided by the animal center of Second Military Medical University.The animals were housed in stainless-steel cages in a room with controlled temperature $\left(22 \pm 2^{\circ} \mathrm{C}\right)$ and humidity $(55 \pm 5 \%)$ and a 12-h light/dark cycle (light: 07:00-19:00 h, dark: 19:00-07:00 h). The animals were fed with standard diet and had free access to water. All experimental procedures were conducted according to the Institutional Animal Care guidelines and approved ethically by the Ethics Committee

*Corresponding author: Heping Xiao, Department of Tuberculosis, Shanghai Pulmonary Hospital, Tongji University School of Medicine, 507 Zhengmin Rd. Shanghai 200433, People's Republic of China, Tel: 86-21-65115006; Fax: 86-2165111298; E-mail: xiaoheping_sars@163.com

Received February 24, 2015; Accepted March 17, 2015; Published March 19 2015

Citation: Yan L, Xie A, Wang Z, Zhang W, Huang Y, Xiao H (2015) Pharmacokinetics of Cycloserine in Rats by HPLC-MS/MS. Med chem 5: 104-107. doi:10.4172/21610444.1000250

Copyright: @ 2015 Yan L, et al. This is an open-access article distributed under the terms of the Creative Commons Attribution License, which permits unrestricted use, distribution, and reproduction in any medium, provided the original author and source are credited. 
of Shanghai Pulmonary Hospital, Tongji University School of Medicine.

\section{Chemicals and reagents}

Cycloserine was supplied by Zhejiang Hisun Pharmaceutical Co. Ltd (China). Pentobarbital was purchased from Sigma-Aldrich Corporation (St. Louis, USA). Ringer's solution was used to perfuse the microdialysis probes and consisted of $145 \mathrm{Mm} \mathrm{NaCl}, 0.6 \mathrm{mM}$ $\mathrm{KCl}, 1.0 \mathrm{mM} \mathrm{MgCl}_{2}$, and $1.2 \mathrm{mM} \mathrm{CaCl}_{2}$ in $2 \mathrm{mM}$ phosphate buffer, $\mathrm{pH}$ 7.4. Methanol (HPLC grade) was purchased from Fisher Scientific (Pittsburgh, PA, USA). Ultrapure HPLC-grade water was prepared using the Milli-Q ${ }^{\bullet}$ system (Millipore, Milford, MA, USA). All other chemicals and reagents were analytical grade from Beijing Chemical Reagent Co. (Beijing, China).

\section{Microdialysis system.}

The microdialysis system consisted of microdialysis pump (CMA402, Swedish CMA company), trace collector (CMA820), CMA/20 microdialysis probes(membrane length of $10 \mathrm{~mm}$ and membrane diameter of $0.5 \mathrm{~mm}$, cutoff of $10 \mathrm{kDa}$, Sweden)and a microliter syringe ( $1 \mathrm{ml}$, gas tight).

\section{Mass spectrometric conditions}

An electrospray ionisation (ESI) source was employed for samples ionisation using the following instrument settings: Curtain gas (CUR) $25 \mathrm{~mL} / \mathrm{min}$, collision-activated dissociation (CAD) medium, the ion spray voltage (IS) 4500 , source temperature (TEM) $550^{\circ} \mathrm{C}$, declustering potential (DP) 25, collision energy(CE)20, collision cell exit potential (CXP) 38,entrance potential (EP)4. Cycloserine was then quantified in multiple-reaction monitoring (MRM) mode using the following mass transitions: $\mathrm{m} / \mathrm{z} 206.9$.

\section{Chromatographic conditions}

The method was developed on a ACE.5C18-AR column (4.6 $\mathrm{mm} \times 150 \mathrm{~mm}, 5 \mu \mathrm{m})$ maintained at $30^{\circ} \mathrm{C}$. The mobile phase was $35: 65$ $(\mathrm{v} / \mathrm{v})$ mixture ofacetonitrile containing $2 \mathrm{mM}$ ammonium formate and $0.1 \%$ aqueous formic acid delivered at a flow rate of $1.0 \mathrm{ul} / \mathrm{min}$. The autosampler was conditioned at $4^{\circ} \mathrm{C}$, and the injection volume was $5 \mathrm{ul}$. Cycloserine was weighed accurately and added into ultra pure water to make a series of solutions. Parallel operated three copies and take $10 \mathrm{u}$ 1 sampling.

\section{Validation of the assay}

Within-run and between-run precisions, linearityand lower limits of detection (LLODs)were evaluated.Within-run precisions were determined by 6 replicated analyses of low, medium and high concentrations of quality control samples. Between-run precisions were determined by measuring low-,medium- and highconcentration controls for 6 consecutive days. Linearity was evaluated as recommended by the CLSI (formerly the NCCLS) [9]. In brief, blank sera and sera spiked with high-concentration standard solutions were mixed in different proportions to produce three solutions containing intermediate concentrations. Each was analysed in quadruplicate. Interassay calibration variability data were evaluated on five consecutive days with concentrations of $5.0-100 \mathrm{mg} / \mathrm{mL}$. The LLOQ was defined as the lowest concentration with a precision $<20 \%$ and an accuracy within $\pm 20 \%$. Under the condition of this experiment, there were no endogenous interfering substances in the dialysate. The within-run precision and the between-run precision was less than $3 \%$ and less than $4 \%$, respectively. The precision and the accuracy comply with the requirements of determination of biological sample. A linear regression was performed between cycloserine area(y) and concentration(X). The standard curve obtained: $y=353.96839 x+504.17744(\mathrm{r}=0.99996)$ (weighting: $1 / \mathrm{x}^{\wedge} 2$ ). It showed that the concentrations of Cycloserine in the range of $22 \mathrm{mg} / \mathrm{l}-2200 \mathrm{mg} / \mathrm{l}$ in the dialysis fluid had good linearity. The shape and resolution of cycloserine was fine. The retention time was $4.6 \mathrm{~min}$. The LLOQ was $22 \mathrm{mg} / \mathrm{l}$.

\section{In vivo recovery}

The in vivo recovery of cycloserine was determined by retro dialysis according to the surgical procedure described below. Rats were fixed in 37 degree Celsius heat insulation pad after anesthetized as previously described [10].An incision was made $3 \mathrm{~cm}$ below the right foreleg, and the thoracic cavities were opened aseptically. The right mean pulmonary lobe was individualized. Microdialysis probe was inserted horizontally through the right mean pulmonary lobe. The vein microdialysis probe was inserted intoinferior vena cava via the left femoral vein.After the insertion, the microdialysis probe and the vein probe were connected to a CMA 402 microdialysis pump. Ringer containing $1 \mathrm{ug} / \mathrm{ml}$ cycloserine was loaded into blood and lung tissue respectively at a constant flow rate of $2 \mathrm{ul} / \mathrm{min}$. Microdialysis samples were collected from each probe at 30-min intervals up to $2 \mathrm{~h}$ and were analyzed immediately. After the samples were collected, all experimental rats were exploratory laparotomy. We did not find obvious pulmonary hemorrhage and abnormal accumulation of fluid. The probes were retained in the lungs for paraffin section to confirm that the site where probe implantation was parenchyma of lung. The drug concentration in the perfusate solution (Cperf) and the drug concentration in the dialysate sample(Cdial) were determined by LC-MS-MS. The in vivo recovery of the probe was calculated by the following formula: Rdial=(Cperf-Cdial)/Cperf. The actual concentration of drugs in vivo $(\mathrm{Cu})$ was obtained by the conversion of continuous monitoring dialysate concentration $(\mathrm{Cm})$ with the formula: $\mathrm{Cu}=\mathrm{Cm} / \mathrm{Rdial}$.

\section{Pharmacokinetic(PK) Study}

Cycloserine was dissolved in sterile saline (SAL, 0.9\% w/v) at concentration of $5 \mathrm{mg} / \mathrm{ml}$ and was administeredby gavage at a dose of $22.5 \mathrm{mg} / \mathrm{kg}$ body weight.Serial blood Samples were collectedright after dosing of cycloserine at 15 -min intervals during the first $240 \mathrm{~min}$ and30-min intervals duringthe last240min. The blood was collected from the fossa orbital is vein and was poured into heparinised tubes. The rat plasma samples were centrifuged (TGL-16G; Shanghai Precision Instruments Co., Ltd., Shanghai, China) at $2500 \mathrm{rpm}$ for $15 \mathrm{~min}$ at $4^{\circ} \mathrm{C}$, and the plasma supernatants were collected. Synchronous monitoring drug concentration changes in the rat pulmonary tissue and blood after administration of $480 \mathrm{~min}$.

Pharmacokinetic data analyses were conducted using Phoenix Win Nonlin software (Pharsight Corporation, Version 6.1,2009) with noncompartment model. The plasma and tissue concentration-versus-time profiles were analyzedto determine the following pharmacokinetic parameters: the area under the concentration-versus-time curve (AUC), the area under the plasma concentration-versus-time curve from zero to last sampling time (AUC 0-t), peakplasma and lung concentration (Cmax), time to reach Cmax (Tmax), final concentration( Clast), final time ( $t$ last), half-time ( $t 1 / 2)$, the first order elimination rate constant from the slope of the log-linear concentration-time regression of data $\operatorname{collected}(z) . \quad A U C=A U C(0-t)+A U C(0-t)=A U C(0-t)+C l a s t / z ;$ The AUC of the first order moment (AUMC) $=$ AUMClast + (tlast $\times$ Clast $/$ $\mathrm{z})+$ Clast/ (z)2. The mean Resident time (MRT)=AUMC/AUC. Bood and lung tissue distribution coefficient of drug was calculated by AUC of lung tissue/AUC of blood. The penetration ratio (PR)of cycloserine to lung tissue was calculated by $\mathrm{C}$ lung /C blood. 


\section{Results}

\section{Pharmacokinetics of cycloserine}

The average recovery rates of blood and lung tissue probe of the six rats were $89.4 \%$ and $86 \pm 5 \%$, respectively. Parameters of noncompartmental model are detailed in Table1.

The concentrations of free cycloserine in blood and lung tissue reached highest at one hour after oral administration, which was 10.613 $\pm 2.423 \mathrm{mg} / \mathrm{L}$ and $1.5266 \pm 0.772 \mathrm{mg} / \mathrm{L}$. And then the concentrations continued to decline. In the monitoring period after administration, concentrations in lung tissue were significantly lower than the concentration in the blood. The area under the concentration-time curve of blood and lung tissue of was $33.532 \pm 6.595 \mathrm{~h} \cdot \mathrm{mg}-1 \cdot \mathrm{L}-1$ and $4.492 \pm 1.081 \mathrm{~h} \cdot \mathrm{mg}-1 \cdot \mathrm{L}-1$. Lung blood barrier permeability was 0.147

\begin{tabular}{|c|c|c|}
\hline parameters & Blood & Lung \\
\hline $\mathrm{t} / \mathrm{2}(\mathrm{h})$ & $2 \pm 1.54$ & $2 \pm 1.79$ \\
\hline $\mathrm{Cmax}(\mathrm{mg} \cdot \mathrm{h} / \mathrm{L})$ & $10.61 \pm 2.42$ & $1.53 \pm 1.71$ \\
\hline $\mathrm{tmax}(\mathrm{h})$ & $1 \pm 0.69$ & $1 \pm 0.58$ \\
\hline AUC0-inf(h-mg-1·L-1) & $33.53 \pm 6.51$ & $4.492 \pm 2.08$ \\
\hline AUC0-t(h.mg-1·L-1) & $33.31 \pm 6.37$ & $4.27 \pm 2.02$ \\
\hline MRT(h) & $3.45 \pm 0.66$ & $3.83 \pm 0.74$ \\
\hline
\end{tabular}

Note: Cmax and tmax was the peak concentration of drug in the tissue and the time reach peak concentration. AUCO-inf and AUCO-t represented the total area under the curve and the area to the end point of observation, respectively. MRT was the average dwell time of the drug molecules in vivo.

Table 1: Pharmacokinetic parameters in rats blood and lung after administration of cycloserine (mean $\pm \mathrm{SD}$ ).

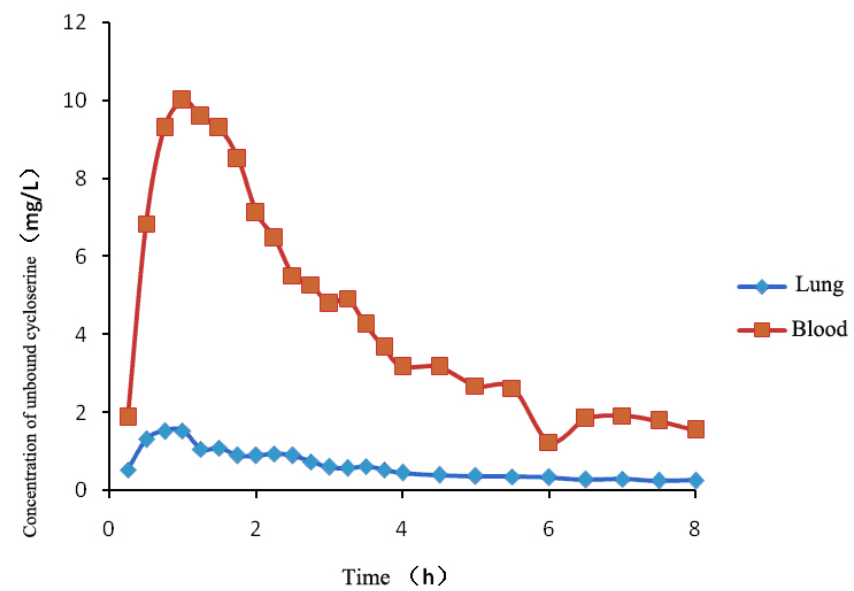

Figure 1:Cycloserine concentration in blood and lung tissues at each sampling

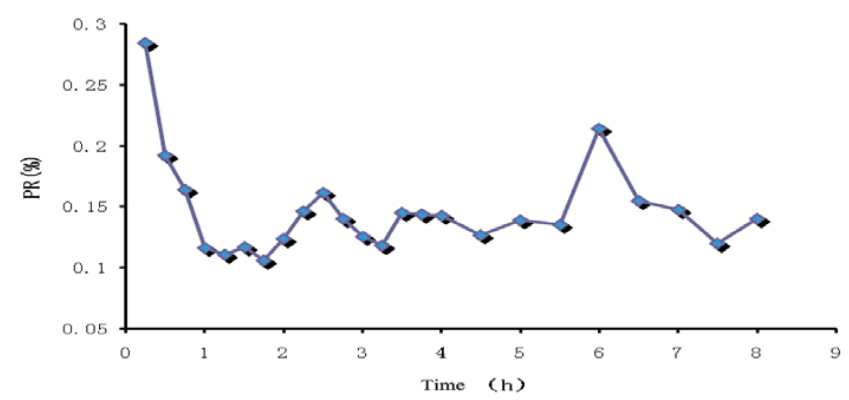

Figure 2: Permeability rate $(P R)$ of lung tissue to blood of cycloserine at all sampling point. \pm 0.039 and the highest and lowest value was 0.285 and 0.105 . The distribution coefficient of cycloserine in blood and lung was 0.134 . Concentrations in blood and lung tissue of cycloserine after intragastric administration at each time point were shown in Figure 1. Lung blood barrier permeability-time curve was shown in Figure 2.

\section{Discussion}

In microdialysis sampling, animal individual differences did not cause significant influence to probe recovery rate. Because the probe used in this experiment was the commercial CMA probe with stable physical property and the selected parts for microdialysis was relatively fixed, the microcirculation transport of drugs in different individuals was similar. The inter-individual differences of measured probe recovery observed is small. In this experiment, the blood and lung probe recoveries were $89 \pm 4 \%$ and $86 \pm 5 \%$, higher than some of the previous microdialysis study $[7,11,12]$. The main reason was small molecule of cycloserine easily through the probe semipermeable membrane, so that the recovery rate increased.

In this experiment, we observe the pharmacokinetics of cycloserine in lung tissue administered $250 \mathrm{mg}$ every time refer to the minimum dosage for human body. The distribution coefficient from blood to lung tissue was $0.15 \pm 0.2$ and the penetration rate from blood to lung tissue has been maintained between 0.28 to 0.10 , most located at about 0.15 . We can see that the penetration rate of cycloserine to lung tissue is poor, probably because cycloserine was small molecular and water soluble substances. We all know that the drug concentration in lung tissue after administration was far lower than the drug concentration in blood (about1/ 30 to $1 / 40$ of concentrations in blood) [13]. While drugs with large molecular and lipid soluble could penetrated into lungs more easily. Rifampicin is an example of this type. Most antibacterial drugs have stronger ability of penetrating inflammatory tissues than normal tissues.The experiment is done on healthy rats. Whether the concentration of cycloserine in lung tissue will be higher when pulmonary inflammation or infection exist or if we administered oral dosing of 500mg every time, the penetration rate would be higher? All these need further descriptive studies.

The half-time of cycloserine in human was $10 \mathrm{~h}$, while in this study the half-time of cycloserine in rats was $2 \mathrm{~h}$. The possible cause was that cycloserine was removed rapidly in rats. Fattorini et al. found that cycloserine was eliminated in a higher rate in mice, significantly faster than in human body [14]. In this study, Cmax in blood was $10.61 \pm 2.42$ $\mathrm{mg} / \mathrm{L}$. It was seen that concentration of cycloserine in blood serine after oral administration was still high.

The bioavailability of cycloserine in human was relatively high. Absorption rate can reach $70-90 \%$ vial oral. Cmax in lung tissue was $10.61 \pm 2.42 \mathrm{mg} / \mathrm{L}$ in this study. In vitro, the minimum inhibitory concentration (MIC) of cycloserine on strain $\mathrm{H} 37 \mathrm{Rv}$ was high $(25$ $\mathrm{mg} / \mathrm{L}$ ) [15]. It is difficult to achieve the desired effect of anti-tuberculosis in the lung tissue. From the view of pharmacodynamics, we suspect that the treatment effect of cycloserine administer $250 \mathrm{mg}$ in oral dosage is limited. Increase the dose was needed. In addition, the clearance rate and the average dwell time of cycloserine in lung tissue and in blood is basically the same which suggest that the elimination process of cycloserine in lung is similar to that in blood.

To kill Mycobacterium tuberculosis often requires combined action of multiple medications. So the treatment effect is very difficult to be judged by single effect of medicines. Treatment of multidrug resistant TB needs of long-term use of drugs more than five kinds. This is bound to the existence of the interactions between drugs. 
Citation: Yan L, Xie A, Wang Z, Zhang W, Huang Y, Xiao H (2015) Pharmacokinetics of Cycloserine in Rats by HPLC-MS/MS. Med chem 5: $104-107$. doi:10.4172/2161-0444.1000250

It was found in the study by David et al that beta-chlorine-dalanine drugs and cycloserine have synergistic effect. The MIC of cycloserine on Mycobacterium tuberculosis can be greatly reduced while combined with beta-chlorine-d-alanine drugs [16]. Many other studies showed scheme containing cycloserine had good results in the treatment of MDR-TB. In Turkey, the cure rate of treatment containing cycloserine in MDR-TB was 77\% [17]. In Japanese, the curative effect of D-cycloserine, ethambutol and pyrazinamide in pregnant MDR-TB patients is remarkable [18]. In Iran, treatment of cycloserine MDR-TB can achieve cure [19]. In India, out of 39 MDR-TB patients receiving the regimen containing cycloserine, 29 (74.3\%) achieved sputum conversion within six months and remained so at the end of two years [20].

Pharmacokinetic data in this body was obtained in health rats. But in practice, the AUC value in infected body often is not the same as in the healthy body. Therefore, in order to obtain the true cycloserine pharmacokinetics more accurately, we need to study further. For example, obtain kinetic parameters in infectious animalsand patients or do experiments on combined administration of multiple antituberculosis drugs. Since lack of theory and experience of MDRTB treatment with cycloserine, we need more laboratory research and clinical practice.

\section{Acknowledgement}

This study was funded by the National Key Project for Infectious Disease (No. 2013ZX10003009). The authors have declared that no competing interests exist.

\section{References}

1. WHO (2014) Global tuberculosis report epidemiology, strategy, financing Geneva, World Health Organization.

2. Bruning JB, Murillo AC, Chacon O, Barletta RG, Sacchettini JC (2011) Structure of the Mycobacterium tuberculosis $D$-alanine:D-alanine ligase, a target of the antituberculosis drug D-cycloserine. Antimicrob Agents Chemother 55: 291 301.

3. Carroll MW, Lee M, Cai Y, Hallahan CW, Shaw PA, et al. (2012) Frequency of adverse reactions to first- and second-line anti-tuberculosis chemotherapy in a Korean cohort. Int J Tuberc Lung Dis 16: 961-966.

4. Mohajeri P, Norozi B, Atashi S, Farahani A (2014) Anti tuberculosis drug resistance in west of iran. J Glob Infect Dis 6: 114-117.
5. Brunner M, Langer O (2006) Microdialysis versus other techniques for the clinical assessment of in vivo tissue drug distribution. AAPS J 8: E263-271.

6. Rowland M, Tozer TN (1995) Concentration Monitoring. In: B alado D ,Klass F , Stead L, Forsyth L, Magee RD , eds. C linical Pharmacokinetics: Concepts and Applications. 3rd ed. Baltimore, MD: Lippincott, Williams \& Wilkins 290-309.

7. Dingde Liu, Heping Xiao, Zhuo Wang (2010) Pharmacokinetics of levofloxacin hydrochloride in rat pancreas. Chinese Journal of Traumatology 10: 93-95.

8. Dhanani J, Roberts JA, Chew M, Lipman J, Boots RJ, et al. (2010) Antimicrobia chemotherapy and lung microdialysis: a review. Int $\mathrm{J}$ Antimicrob Agents 36: 491-500.

9. National Committee for Clinical Laboratory Standards (2003) Evaluation of the Linearity of Quantitative Measurement Procedures: A Statistical Approach: Approved Guideline EP6-A. NCCLS, Wayne, PA, USA.

10. Giachero M, Bustos SG, Calfa G, Molina VA(2013)ABDNF sensitive mechanism is involved in the fear memory resulting from the interaction between stress and the retrieval of an established trace. Learn Mem 20: 245-255.

11. Tasso L, Bettoni CC, Oliveira LK, Dalla Costa T (2008) Evaluation of gatifloxacin penetration into skeletal muscle and lung by microdialysis in rats. Int J Pharm 358: 96-101.

12. Plock N, Kloft C (2005) Microdialysis--theoretical background and recent implementation in applied life-sciences. Eur J Pharm Sci 25: 1-24.

13. Manying Zhang, Jingli Yang (2000) Pharmacokinetics of antibacterial drugs in lung. Chinese Journal of rural doctor 10: 18.

14. Fattorini L, Tan D, Iona E, Mattei M, Giannoni F, Brunori L, et al. (2003) Activities of moxifloxacin alone and in combination with other antimicrobial agents against multidrug-resistant Mycobacterium tuberculosis infection in BALB/c mice. Antimicrob Agents Chemother 47: 360-362.

15. [No authors listed] (2008) Cycloserine. Tuberculosis (Edinb) 88: 100-101.

16. David S (2001) Synergic activity of D-cycloserine and beta-chloro-D-alanine against Mycobacterium tuberculosis. J Antimicrob Chemother 47: 203-206.

17. Tahaoglu K, Torun T, Sevim T, Atac G, Kir A, et al. (2001) The treatment of multidrug-resistant tuberculosis in Turkey. N Engl J Med 345: 170-174.

18. Takashima T, Danno K, Tamura Y, Nagai T, Matsumoto T, et al. (2006) [Treatment outcome of patients with multidrug-resistant pulmonary tuberculosis during pregnancy]. Kekkaku 81: 413-418.

19. Masjedi MR, Tabarsi P, Chitsaz E, Baghaei P, Mirsaeidi M, et al. (2008) Outcome of treatment of MDR-TB patients with standardised regimens, Iran 2002-2006. Int J Tuberc Lung Dis 12: 750-755.

20. Prasad R, Verma SK, Sahai S, Kumar S, Jain A (2006) Efficacy and safety of kanamycin, ethionamide, PAS and cycloserine in multidrug-resistant pulmonary tuberculosis patients. Indian J Chest Dis Allied Sci 48: 183-186. 